\title{
A mixed methods service evaluation of a pilot functional electrical stimulation clinic for the correction of dropped foot in patients with chronic stroke
}

\author{
Jane Shiels ${ }^{1}$, Katie Wilkie ${ }^{1}$, Cathy Bulley ${ }^{2}$, Stephen Smith ${ }^{3}$ and Lisa Salisbury ${ }^{4}$ \\ ${ }^{1}$ Specialist Physiotherapist, Physiotherapy Department, Astley Ainslie Hospital, Edinburgh, Scotland, UK \\ ${ }^{2}$ Lecturer in Physiotherapy, Queen Margaret University, Musselburgh, East Lothian, Scotland, UK \\ ${ }^{3}$ Consultant in Rehabilitation Medicine, Astley Ainslie Hospital, Edinburgh, Scotland, UK \\ ${ }^{4}$ Research Fellow, University of Edinburgh, Edinburgh, Scotland, UK
}

\begin{abstract}
Aim: To undertake a service evaluation of the pilot Lothian functional electrical stimulation (FES) clinic using both quantitative and qualitative methods and clinical practice reflection. Background: Clinical guidelines recommend that FES, for the management of dropped foot after stroke, is delivered by a specialist team. However, little detail is provided about the structure and composition of the specialist team or model of service delivery. A pilot Lothian FES clinic was developed to explore the clinical value of providing such a service to stroke patients with dropped foot and identify any service modifications. Methods: Mixed methods were used to evaluate the service and included quantitative, qualitative and reflective components. Phase 1: Before and after service evaluation of patients attending the FES clinic between 2003 and 2007. Outcomes of gait velocity and cadence were recorded at initial clinic appointment and 6 months after application of FES. Phase 2: Qualitative research exploring patients with stroke and carers' experiences of the FES clinic. Data were collected via semi-structured interviews. Phase 3: A reflection on the service delivery model. Participants: Phase 1: 40 consecutive out-patients with stroke; Phase 2: 13 outpatients with stroke and 9 carers; Phase 3: Three specialist physiotherapists engaged in running the FES clinic. Findings: Statistically significant improvements $(p<0.001)$ were demonstrated in gait velocity and cadence. Qualitatively, one super-ordinate theme 'The FES clinic met my needs' emerged. Within this were four sub-themes, namely 1. 'Getting to grips with FES wasn't difficult'; 2 . 'It's great to know they're there'; 3. 'Meeting up with others really helps' and 4. 'The service is great but could be better'. On reflection, minor modifications were made to the service delivery model but overall the service met user needs. This dedicated FES clinic produced positive physical outcomes and met the needs of this chronic stroke population.
\end{abstract}

Key words: dropped foot; functional electrical stimulation; physiotherapy; service evaluation; stroke

Received 21 January 2010; accepted 12 January 2011

\section{Introduction}

Correspondence to: Jane Shiels, Specialist Physiotherapist, Physiotherapy Department, Astley Ainslie Hospital, 133 Grange Loan, Edinburgh, Scotland, EH9 2HL, UK. Email: Jane.Shiels@nhslothian.scot.nhs.uk
Stroke affects between 174 and 216 people per 100000 population in the United Kingdom (Royal College of Physicians, 2008). Of those, it is

C) Cambridge University Press 2011 
estimated that $18 \%$ of patients will present with a dropped foot (Verdie et al., 2004). Functional electrical stimulation (FES) is the application of electrical stimulation to produce a functional movement. One commonly reported use of FES is as an orthotic device for the correction of dropped foot during walking. Stimulation is provided via skin surface or implanted electrodes and triggered by a pressure-sensitive footswitch worn inside the shoe and attached to the FES device. The device activates the dorsiflexors, causing the foot to lift, during the swing phase of gait.

Evidence supporting the efficacy of FES in a chronic stroke population has emerged in recent years (Taylor et al., 1999a; 1999b; Burridge, 2001; Kottink et al., 2004; Robbins et al., 2006; Laufer et al., 2009; National Institute for Health and Clinical Excellence, 2009). This research has provided evidence of the positive benefits of the application of FES, namely reduction in energy expenditure, increased gait velocity, decreased falls and improved quality of life (Taylor et al., 1999a; 1999b; Burridge, 2001; Kottink et al., 2004; Robbins et al., 2006; Laufer et al., 2009; National Institute for Health and Clinical Excellence, 2009). The National Guidelines for Stroke propose that FES should only be considered when an ankle foot orthosis (AFO) does not adequately control the dropped foot and when obvious gait improvements can be demonstrated (Royal College of Physicians, 2008). This recommendation may in part be due to successful historical management of dropped foot using orthotic splints, for example, AFO. However, more recently, NICE (the National Institute for Health and Clinical Excellence) produced interventional procedural guidance stating that the evidence on the safety and efficacy of FES in relation to gait supports its clinical application with the proviso that normal clinical governance activities occur, for example, audits (National Institute for Health and Clinical Excellence, 2009). Despite the evidence base and these recommendations, dedicated FES services are not widely available throughout the United Kingdom (National Institute for Health and Clinical Excellence, 2009). Anecdotal evidence suggests that effective delivery of FES within routine practice is complex and that compliance from patients is poor. The National Guidelines for Stroke propose that FES should only be applied by a specialist team able to evaluate its benefits beyond the current management strategies, although the rationale for this or the definition of what comprises a 'specialist team' is not reported (Royal College of Physicians, 2008).

Limited literature exists documenting in detail FES clinical service provision and perception of service users. A service review by Taylor et al. (1999a) in 1999 concluded that their service met the needs of past and present users. Background information was provided on the number of patient appointments, follow-up procedure and inclusion/ exclusion criteria of their service. However, clear information on appointment length, composition and skill mix of professionals involved and levels of staffing was not reported. Service users' perceptions of the value of FES and satisfaction with the clinical service were collected by the clinical team, using a closed questionnaire, on diverse mixed pathologies, with a response rate of $55 \%$ (Taylor et al., 1999a). The majority of service users perceived that the clinical team provided good service with value being placed on the explanation and written information provided. However, the method of data collection, mixed pathologies, poor response rate and lack of service provision detail limit the clinical transferability of these results to a stroke population. Clarification of the 'specialist team', optimum service design and the clinical benefits of such a service in stroke is still required.

Following clinical training in FES, a Lothian pilot outpatient clinic was designed and commenced in 2003. The clinic model was based on the available evidence base (Taylor et al., 1999a) and contact with an established clinical service, the National Clinical FES Centre, Salisbury (National Clinical FES Centre). The National FES Centre is a full-time dedicated clinical service accepting referrals from throughout the United Kingdom. Their service delivery model was specific to the needs of their patient group, included patients from outside their locality and was staffed by physiotherapists and bioengineers. The Lothian service delivery model was designed to accommodate the local population, staffed entirely by physiotherapists and formed one component of existing neurological physiotherapy outpatient services. At its concept, and in line with good clinical practice and governance, systematic service evaluation was a core component of the pilot study to establish feasibility and effectiveness of the service. Although quantitative service evaluation may produce positive 
objective physical outcomes, the service may still fail to meet the needs of service users and be too complex to deliver clinically. Current Government stroke strategy recommends that when developing services, people with stroke and their carers are meaningfully involved in service provision, delivery and monitoring (Department of Health, 2007). By its nature, qualitative research aims to explore, understand and conceptualise a specific phenomenon from the perspective of those directly experiencing it. This approach can be used to systematically secure and explore the views of service users and could be considered as an option when carrying out service evaluation. The addition of the reflections of those delivering the service is clinically valuable in ascertaining the possible issues in relation to its clinical provision.

\section{Aims}

To undertake a service evaluation of the pilot Lothian FES clinic, using both quantitative and qualitative methods, to analyse and explore the service design, specialist team and clinical benefits. To reflect on the clinical issues of delivering such a service.

\section{Methods}

The Lothian FES pilot clinic was funded for a limited number of patients, between 2003 and 2007 , by Ecas, a local charity, and the Lothian Managed Clinical Network (MCN) for Stroke. The FES device provided was the single channel Odstock Dropped Foot Stimulator (ODFS), a licensed medical device available from Odstock Medical Limited, Salisbury, United Kingdom. The clinic was set up and run by three specialist neurological physiotherapists trained in the application of FES. This specialist team identified inclusion criteria for access to the clinic (Table 1) and designed a service delivery model (Figure 1). The service was designed to provide intensive clinical input in the initial stages post set-up. Patients received annual reviews to monitor progress and equipment set up from six months onwards. Outside formal appointments, patients could contact the service for consumables (spare leads, footswitches and electrode pads) and telephone advice as required. In addition to this, all service users
Table 1 FES pilot clinic service inclusion/exclusion criteria

\begin{tabular}{|c|c|}
\hline Inclusion & Exclusion \\
\hline $\begin{array}{l}\text { Foot drop secondary to } \\
\text { upper motor neurone } \\
\text { lesion }\end{array}$ & $\begin{array}{l}\text { Severe increased tone in } \\
\text { calf }\end{array}$ \\
\hline $\begin{array}{l}\text { Able to stand unsupported } \\
\text { and walk minimum of } \\
10 \text { m (can be with an aid, } \\
\text { but not assistance) }\end{array}$ & $\begin{array}{l}\text { Loss of range at ankle joint of } \\
\text { greater than } 10 \text { degrees off } \\
\text { plantar grade } \\
\text { Poor skin condition }\end{array}$ \\
\hline $\begin{array}{l}\text { Motivation to use the } \\
\text { device }\end{array}$ & Pacemaker \\
\hline $\begin{array}{l}\text { Ability to understand } \\
\text { instructions for use }\end{array}$ & Pregnancy \\
\hline $\begin{array}{l}\text { No other medical } \\
\text { condition severe enough } \\
\text { to impair walking }\end{array}$ & $\begin{array}{l}\text { Severe lower limb oedema } \\
\text { Uncontrolled epilepsy }\end{array}$ \\
\hline
\end{tabular}

$\mathrm{FES}=$ Functional electrical stimulation .

including carers were invited to attend an annual users group meeting. This was an informal gathering giving patients and carers the opportunity to share information and for staff to update users on the service and any developments. Initially, clinic referrals were received from Lothian physiotherapists. As the service became more established, referrals were received from other health professionals, for example, consultants, specialist nurses.

Three phases of evaluation were undertaken. Phase 1 was a quantitative analysis of clinical outcomes, Phase 2 was an exploration of patient perceptions of the service and Phase 3 was a reflective review of the service delivery model conducted by the clinical team.

\section{Phase 1}

As this was a service evaluation and the data collected were part of routine practice, no ethics approval was required (National Patient Safety Agency, 2009).

\section{Study design}

A before and after service evaluation was undertaken. Routine data collected at initial assessment and six months post-application of FES were analysed. Outcomes were gait velocity $(\mathrm{m} / \mathrm{s})$ and cadence (steps per minute) measured during a timed $10-\mathrm{m}$ walk. Such measures have robust psychometric properties (Mudge and Stott, 2007). Demographic details were collected. 


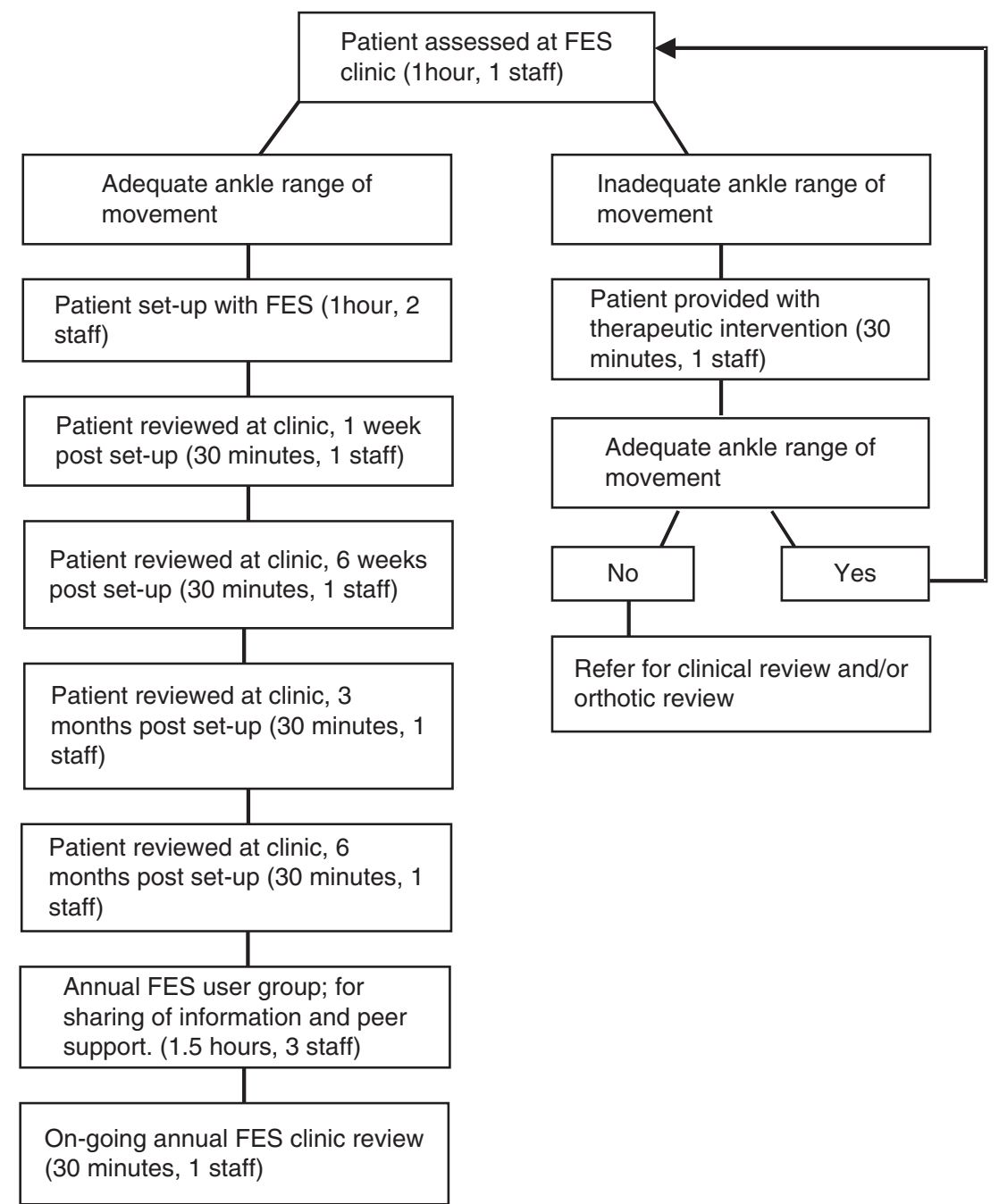

Figure 1 FES pilot clinic service delivery model

\section{Participants}

Owing to the nature of the funding, patient numbers attending the FES clinic were restricted to a maximum of 50 , with the majority having a diagnosis of stroke. A limited number, three, had progressive illnesses, for example, multiple sclerosis (MS) requiring more intensive service provision. In addition, owing to the small number of patients with progressive diagnosis and the nature of their illness, a pragmatic decision was taken to exclude them from this evaluation. A number of patients with stroke dropped out for a variety of reasons; one was lost to follow-up; two were not suitable on assessment (inadequate range of movement); one died; two were unable to tolerate the sensation of the device; and one no longer required FES due to recovery of movement. Therefore, the service evaluation was conducted on a convenience sample of 40 consecutive patients with stroke who had attended the Lothian FES clinic for at least six months between 2003 and 2007. Prior to attendance at the clinic, some patients routinely used an AFO while others used no orthotic device. Analysis was 
undertaken in both the whole group and these identified subgroups to evaluate whether there were any differences in the effectiveness of FES.

\section{Analysis}

Prior to detailed analysis, the data were assessed and confirmed to have normal distribution. Therefore, descriptive means and standard deviations were used. A paired sample $t$-test was used for the analysis of the total cohort results between the two time-points (baseline and six months). In addition, subgroup analysis using an independent sample $t$-test was undertaken to compare differences between subgroups (patients who routinely used an AFO versus those who used no orthotic device prior to the application of FES).

\section{Phase 2}

The qualitative component of this study was not part of the routine clinical data collection and therefore ethics approval was sought and granted from the Lothian Research Ethics Committee 1 Ref. No. 06/S1101/37.

\section{Study design}

A qualitative phenomenological research study was undertaken between April and May 2007. Purposive sampling with an agreed stratification process was used. Stratification of participants was based on time since stroke and gait velocity at initial FES set-up. Data were collected using face-to-face semi-structured interviews undertaken by an independent researcher (CB). The independent researcher was an experienced qualitative researcher who had no involvement in the FES clinic. Owing to this data collection method, patients with severe communication difficulties were excluded. In recognition of this, carers of patients with stroke, who had severe communication difficulties and had attended the clinic, were actively sought for inclusion. An interview schedule (Table 2) based on the literature and Phase 1 evaluation was developed by the research team comprising two specialist physiotherapists and two research academics (JS; $\mathrm{KW}$; LS; CB). The interview schedule reflected the aim of the study and was deliberately broad in nature to allow participants the opportunity to elaborate. Interviews were digitally recorded and undertaken at a venue of the participant's choice. All interviews were transcribed verbatim and
Table 2 Phase 2: qualitative interview schedule

Interview schedule

How did you find learning to use it?

Now you have been using FES for more than six months, what support do you receive?

How does that affect you?

How much support would you like to receive?

Is there anything you feel you need that you did not receive at the moment?

Were there any particular negative things about it that stick out in your mind?

Were there any particular positive things about it that stick out in your mind?

FES $=$ Functional electrical stimulation

anonymised by a research assistant. Participant interview summary verification was used.

\section{Participants}

Participants were invited to participate via an introductory letter and information sheet sent by post. Those wishing to participate were asked to contact the researcher directly. The interviews were undertaken at a time and place of the participant's choosing. Prior to commencement of the interviews, written consent was gained. Information was sent to fifteen patients with stroke and eight carers. Of those, thirteen patients and seven carers agreed to participate. A further two carers present at the time of patient interviews consented to have their views included. In total, thirteen patients with stroke and nine carers who had been attending the Lothian FES clinic for at least six months were recruited.

\section{Analysis}

The qualitative element of this study was thematically analysed using the framework of interpretative phenomenological analysis (Smith, 2008). Interpretative phenomenological analysis is a recognised phenomenological-based approach that can be applied to qualitative research. It follows an idiographic approach to analysis, taking each case as unique and from that seeks to find connections or patterns (Smith, 2008). Analysis was undertaken by an independent researcher (CB). Transcripts were read and re-read and emergent themes identified. Themes were constantly reviewed and checked to ensure that they reflected the participant's thoughts. Emergent themes and 
Table 3 Phase 1: quantitative demographic details

\begin{tabular}{llll}
\hline Patient characteristics & $\begin{array}{l}\text { Total cohort } \\
(n=40)\end{array}$ & $\begin{array}{l}\text { AFO subgroup } \\
(n=24)\end{array}$ & $\begin{array}{l}\text { No orthotic subgroup } \\
(n=16)\end{array}$ \\
\hline $\begin{array}{l}\text { Sex (male/female) } \\
\text { Age, years (mean } \pm \text { SD) }\end{array}$ & $28 / 12$ & $14 / 10$ & $14 / 2$ \\
Side of stroke (left/right) & $60 \pm 11.37$ & $59.2 \pm 12.07$ & $55.4 \pm 9.95$ \\
$\begin{array}{l}\text { Time, years since stroke to FES set-up } \\
\quad \text { (mean } \pm \text { SD) }\end{array}$ & $19 / 21$ & $14 / 10$ & $5 / 11$ \\
\hline
\end{tabular}

AFO = ankle foot orthosis; FES = Functional electrical stimulation.

codes were discussed, verified and agreed by the research team. Agreed codes were then applied to all transcripts and connections sought.

\section{Phase 3}

Following the quantitative and qualitative components of the service evaluation, the clinical team, comprising three specialist physiotherapists, who devised and managed the clinical delivery of the service undertook a period of reflection and re-evaluation of the service delivery model. This clinical practice reflection applied no specific methodological approach and aimed to review the staffing skill mix, length and time frame of appointments, patient population and modifications to the service required.

\section{Results}

\section{Phase 1 (quantitative)}

The demographic details of 40 patients with stroke evaluated are presented in Table 3. Of the total cohort, 24 patients were routinely using an AFO at initial contact with the clinic (AFO subgroup). Despite having a dropped foot, the remaining 16 chose not to use an orthotic device (no orthotic subgroup). Table 3 also includes the demographics of these two subgroups.

Results on the total cohort demonstrated that there were statistically significant improvements $(P<0.001)$, in both gait velocity and cadence between baseline and six months, indicating that overall patients walked faster and were able to take more steps. Similarly, within both subgroups (AFO subgroup and no orthotic subgroup), there were statistically significant improvements in gait velocity (AFO subgroup $P<0.001$; no orthotic subgroup $P<0.005$ ) and cadence (AFO sub- group $P<0.005$; no orthotic subgroup $P<0.01$ ). Between subgroup analysis revealed that there were statistically significant differences in gait velocity both at baseline $(P=0.004)$ and at six months $(P<0.001)$, with the patients in the no orthotic subgroup performing faster at both time points. In contrast, there was no statistically significant difference between the subgroups for cadence at baseline $(P=0.199)$. However, at six months, there was a statistically significant difference between subgroups $(P=0.021)$, with the patients in the no orthotic subgroup taking more steps. A summary of these results is presented in Table 4.

As of August 2010, 42.5\% $(n=17)$ of the total cohort continue to use FES. Of those no longer using FES, $17.5 \%(n=7)$ ceased use due to a perceived lack of benefit; $12.5 \%(n=5)$ no longer required FES or any orthotic device due to motor recovery; $12.5 \%(n=5)$ have died; $7.5 \%(n=3)$ had a medical deterioration; and $7.5 \% \quad(n=3)$ were lost to follow-up.

\section{Phase 2 (qualitative)}

Consent was obtained from thirteen patients with stroke and nine carers. Participant characteristics and demographic details are presented in Table 5. In summary, patients with stroke, including those being cared for by interviewed carers, had a mean (SD) age of 64.3 (9.48) years, on average (SD) were 4.63 (1.93) years poststroke and the average (SD) time since set-up with FES was 2.37 (1.01) years. The average (SD) length of interviews was 44.3 (14.5) minutes.

The super-ordinate theme that emerged from the rich data was 'The Lothian FES clinic met my needs'. From within this, four sub-themes were identified (see Figure 2). 
Table 4 Phase 1: quantitative results

\begin{tabular}{lccc}
\hline & Baseline & Six months post set-up with FES & Mean improvement \\
\hline $\begin{array}{l}\text { Total cohort }(n=40) \\
\quad \text { Gait velocity }\end{array}$ & $0.51 \pm 0.2$ & $0.71 \pm 0.33$ & $0.20 \pm 0.23^{*}$ \\
$\quad \begin{array}{l}\text { Cadence } \\
\text { AFO subgroup }(n=24)\end{array}$ & $75.3 \pm 18.5$ & $86.2 \pm 21.9$ & $10.98 \pm 15.77^{*}$ \\
$\quad$ Gait velocity & $0.46 \pm 0.17^{* *}$ & $0.59 \pm 0.23^{* *}$ & $0.13 \pm 0.13^{*}$ \\
$\quad$ Cadence & $72.16 \pm 17.9$ & $79.01 \pm 20.54^{* *}$ & $6.83 \pm 10.84^{*}$ \\
No orthotic subgroup $(n=16)$ & $0.63 \pm 0.22^{* *}$ & $0.96 \pm 0.33^{* *}$ & $0.33 \pm 0.30^{*}$ \\
$\quad$ Gait velocity & $79.90 \pm 18.83$ & $95.68 \pm 21.73^{* *}$ & $15.78 \pm 21.32^{*}$ \\
Cadence &
\end{tabular}

FES $=$ Functional electrical stimulation.

All values are given in mean and SD.

* Statistically significant differences within groups.

**Statistically significant differences between subgroups.

Each sub-theme will be reported in turn.

1. 'Getting to grips with FES wasn't difficult'.

Participants reflected on the learning process on the application of FES. Both carers and patients with stroke indicated, that to them, it was not difficult to learn.

I think it was quite simple. Once you got the spot to put it in. You had to get it in the right place and I find it quite simple. I found it much easier than putting a splint on wae (with) all these straps.

(Moira, carer)

No problem. They gave us a photograph of where to stick it.

(David, patient)

For some, this ease was perhaps related to the faith and confidence that participants had in the staff running the clinic and the perceived expertise and knowledge that they had.

Easy. No problem at all. Again, Elaine, Jean and the others were very helpful.

(Colin, patient)

I was quite impressed, well I got the impression they knew what they were doing.

(Aaron, carer)

2. 'It's great to know they're there'.

All participants placed value on the support and ease of access provided by the clinic and its staff.
It's just great to know that it's there, to know that there's somebody to speak to or contact.

(Keith, patient)

I know that if we need it we can phone up, the service is there.

(Alison, carer)

Accessing spares and consumables for the FES device was not considered to be an issue.

If I'm needing footswitches or the things for your leg (electrode pads), I just usually go in, I just go in and I see them, and they just gae (give) me some.

(Kevin, patient)

Yeah, and if I need pads I just phone in and they post them out.

(Marie, carer)

3. 'Meeting up with others really helps'.

The participants placed great value on user groups and the ability to meet up with other people in their situation. It was felt to be a great opportunity to share information and to keep up to date with the service and developments in the technology.

Well they had a regular series of meetings at the hospital where all the people came that were involved or who had the FES thing, came along to the meeting and were able to share their opinions and their views on it. And that was very helpful, very sort of meaningful in 
Table 5 Phase 2: qualitative research participant characteristics

\begin{tabular}{|c|c|c|c|c|c|c|}
\hline Pseudonyms & $\begin{array}{l}\text { Patient age } \\
\text { (years) }\end{array}$ & Stroke & $\begin{array}{l}\text { Years since } \\
\text { stroke }\end{array}$ & $\begin{array}{l}\text { Timed } 10-\mathrm{m} \\
\text { walk at set-up } \\
\text { (seconds) }\end{array}$ & $\begin{array}{l}\text { Time since } \\
\text { FES set-up } \\
\text { (years) }\end{array}$ & Participants social background \\
\hline Keith (patient) & 44 & $\begin{array}{l}\text { Right basal ganglia } \\
\text { bleed }\end{array}$ & 4 & 13 & 4 & Married, back working \\
\hline Kevin (patient) & 60 & Left TACS & 2 & 15 & 1 & $\begin{array}{l}\text { Lives with relatives, retired due to } \\
\text { stroke }\end{array}$ \\
\hline Paul (patient) & 68 & Left MCA infarct & 3 & 17 & 2 & Married, back working \\
\hline Matthew (patient) & 66 & Left hemiplegia & 9 & 15 & 1 & Married, retired due to stroke \\
\hline David (patient) & 67 & Left hemiplegia & 5 & 14 & 2 & Married, retired \\
\hline Steven (patient) & 61 & Left MCA infarct & 3 & 31 & 3 & Lives with partner, back working \\
\hline Lliam (patient) & 61 & $\begin{array}{l}\text { Right internal capsule } \\
\text { infarct }\end{array}$ & 6 & 23 & 2 & Lives with son. Retired due to stroke \\
\hline Christopher (patient) & 79 & Bilateral infarcts & 7 & 17 & 4 & Married, retired \\
\hline Luke (patient) & 43 & Left TACS & 2 & 24 & 1 & $\begin{array}{l}\text { Divorced with children, remarried } \\
\text { after stroke, back working }\end{array}$ \\
\hline Colin (patient) & 75 & Left lacunar infarct & 6 & 23 & 4 & $\begin{array}{l}\text { Married to Alison, retired due to } \\
\text { stroke }\end{array}$ \\
\hline Aaron (carer) & 57 & Left ICH & 5 & 16 & 3 & Married, both work \\
\hline Daniel (carer) & 65 & Left hemiplegia & 5 & 33 & 2 & Married, retired to look after his wife \\
\hline Marie (carer) & 69 & Left TACS & 7 & 20 & 2 & Married, retired \\
\hline Shona (carer) & 58 & Left TACS & 2 & 16 & 2 & $\begin{array}{l}\text { Married with young children, now } \\
\text { part-time due to husband's stroke }\end{array}$ \\
\hline Lisa (carer) & 67 & Left MCA infarct & 3 & 72 & 2 & Married, retired \\
\hline Alison (carer) & 75 & Left lacunar infarct & 6 & 23 & 4 & $\begin{array}{l}\text { Married to Colin, retired to look after } \\
\text { husband }\end{array}$ \\
\hline $\begin{array}{l}\text { Jack (patient) } \\
\text { Moira (carer) }\end{array}$ & 71 & Right PACS & 6 & 17 & 2 & Married, both retired \\
\hline $\begin{array}{l}\text { Donald (patient) } \\
\text { and Fiona (carer) }\end{array}$ & 73 & $\begin{array}{l}\text { Multiple strokes } \\
\text { affecting left }\end{array}$ & 3 & 31 & 2 & Married, both retired \\
\hline $\begin{array}{l}\text { Euan (patient) } \\
\text { and Anne (carer) }\end{array}$ & 64 & $\begin{array}{l}\text { Left TACS secondary } \\
\text { to haemorrhage }\end{array}$ & 4 & 14 & 2 & Married, retired due to stroke \\
\hline
\end{tabular}

TACS = total anterior circulation stroke; $\mathrm{MCA}=$ middle cerebral artery; $\mathrm{ICH}=$ intracranial haemorrhage; $\mathrm{PACS}=$ partial anterior circulation stroke. 


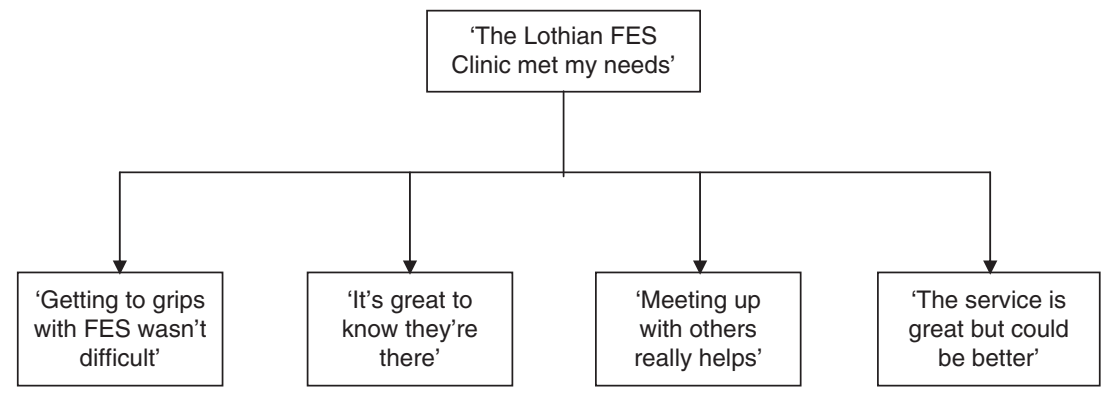

Figure 2 Phase 2: super-ordinate and sub-themes

some way because it kept you in touch, it kept you aware of you know, what was happening.

(David, patient)

They do have meetings every so often where, you know, everybody with the FES goes along and you discuss, you know, what you're finding and people come up with different ideas.

(Alison, carer)

4. 'The service is great but could be better'

Although it was felt to be a good service, some participants identified areas that they felt could be improved. This related to time between reviews and increased number of user groups per annum.

I mean it's a year, we go in yearly now just to check up, but no I feel three months.

(Paul, patient)

I think if we were seen twice a year it would help because having read the literature there's another two things I'd like to know about and I don't like to phone them up because obviously they're very busy, so yes I think there should be a question and answer period twice a year.

(Daniel, carer)

Although not specifically identifying it as a direct service provision issue, Shona recalled selfmanaging a problem that had occurred in relation to equipment testing.

I wrote down the instructions of how to test the footswitch so if that happens again I'll know how to do it.

(Shona, carer)
Potentially, this is not an isolated problem and in the future could be addressed by a simple clinic information leaflet provided to service users.

\section{Phase 3}

Results

The clinical team (three specialist physiotherapists) undertaking the clinical practice reflection were all specialist neurological physiotherapists, with on average (SD) 17 (2.6) years' experience in neurology, trained in the application of FES and directly involved in the development, delivery and evaluation of the service.

\section{Service delivery and staffing}

As FES is a treatment modality relating to gait rehabilitation and is an area in which physiotherapists have recognised expertise and training, it was felt that an FES clinic sat naturally within a neurological physiotherapy service. It was identified that specialist knowledge and expertise in the management and treatment of patients with stroke were beneficial. For clinicians involved in the application of FES in stroke populations, clinical experience is invaluable in recognising and understanding gait abnormalities demonstrated by such patients and identifying those who would benefit from FES. The Lothian service staffing level was based on the National FES centre which had two clinical staff members at set-up of the device. The clinical team adopted this model but recognised that this was a labourintensive component of the service. On reflection, the team felt that the use of two staff members at set-up remained an appropriate use of time. However, as the team became more experienced, 
the length of time for set up appointments reduced. In addition, with increasing experience, more efficient time management strategies were introduced where two new patients could be set up in tandem. Tandem set-up provided additional clinical support, if required, and opinion for the set-up of more complex patients. The clinic was easy to fit into established services. In addition, the time frame for appointments, including the ongoing annual review, was felt to be appropriate and met the needs of a stroke population. This perception was reinforced by the high compliance rate seen with a dropout rate of those deemed suitable for FES of only $5 \%$.

\section{Patient population}

The criteria resulted in appropriate and suitable stroke patients being referred to the clinic. The majority of patients seen at the clinic were patients with chronic stroke. These patients were appropriate for FES and due to the static nature of their illness were overall relatively straightforward to set up and support.

\section{Service modifications}

The service delivery model was easy to deliver clinically, perceived to meet the needs of the stroke population and no direct modifications to the service delivery model were felt necessary, although with experience the clinic became more time efficient. The users group meetings were perceived by clinical staff to be of limited clinical value and potentially one component of the service that could be removed, but following patient and carers feedback the users group meetings have been retained. The team recognised that the clinical service provided a large volume of information to patients and carers at set-up and have therefore begun producing additional information leaflets to support the clinic.

\section{Discussion}

The results from this service evaluation demonstrated that the Lothian FES pilot clinic provided a beneficial service to patients after stroke. Statistically significant improvements in gait velocity and cadence were demonstrated, indicating a functional improvement with FES. Surprisingly, patients who already had an orthotic device at initial assessment still demonstrated statistically significant improvements in physical outcomes using FES, although this was less than those with no orthotic device at the time of assessment. From the qualitative data, patients and carers perceived that the clinic met their needs, but were able to propose future improvements to the service. In addition, the team perceived that the service delivery model met the needs of the FES clinic users, was appropriate for this population and was straightforward to deliver.

The statistically significant improvements in gait velocity in this service evaluation were in line with previous research (Taylor et al., 1999a; Robbins et al., 2006). This would indicate that the inclusion/exclusion criteria were targeted at an appropriate stroke population. In addition, in this evaluation, both subgroups demonstrated significant improvements in velocity and cadence indicating that patients with stroke who previously used an $\mathrm{AFO}$ and those who used no device have the potential to benefit from the application of FES. This model of service delivery has provided significant benefits. This would suggest that quantitatively the clinic model is effective and should continue to be delivered in this way. The National Guidelines for Stroke propose that FES should only be applied by a specialist team able to evaluate its benefits beyond the current management strategies (Royal College Physicians, 2008). This evaluation offers possible clarity over what comprises a 'specialist team' and proposes service delivery and intervention evaluation methods. Future evaluation of the cost effectiveness of this clinic, through health economic analysis, would be of value in establishing the long-term financial implications.

Qualitative research was undertaken to encompass the perceptions and experiences of service users. Overall, they reported that the FES clinic met their needs and that they valued the ongoing support provided by the specialist team. The education regarding the FES device, provided by the clinic, was perceived by service users as appropriate and uncomplicated. Anecdotal evidence would indicate that compliance and long-term use is difficult without good education and ongoing support as offered by a dedicated clinic. However, there is no evidence comparing dedicated FES clinics with routine clinical application of the device. Research exploring this area would be 
of benefit. Prior to the qualitative component of this service evaluation, the users group meetings were viewed by the clinical team to be of limited value and consideration was being given to their cessation. However, patients and carers believed these groups to be of great personal value, describing the benefits that such meetings brought, for example, sharing of experiences, improved knowledge on the use of the device. As a result of this qualitative study, the users group meetings have continued to be an integral component of the service. Another comment raised in relation to the clinic was the desire for increased number of clinic visits beyond the six-month period. While potentially of value to service users, this has additional cost implications. It remains unclear what added benefit to the overall outcome this could bring and therefore requires further investigation. One participant reported an issue regarding equipment testing. This is unlikely to be an isolated issue and has drawn attention to the need to address it. This could be readily resolved by an information leaflet.

The clinical practice reflection component of this service evaluation provides anecdotal but clinically valuable information regarding the possible issues in delivering this clinic as a mainstream service within routine clinical practice. The clinic met the needs of a specific population, namely chronic stroke. Chronic patients with stroke tend to have an established static neurological presentation and could be perceived as less complex to set up with FES. This service delivery model may not be appropriate or meet the needs of patients with progressive or complex neurological problems, such as MS or incomplete spinal cord lesions. These groups may require more clinical input and support. Further service evaluation with differing neurological populations is warranted. Although the service delivery model has not been directly modified, more efficient use of initially set up appointments has been implemented. This is only possible due to the increased clinical experience in the application of FES by the clinical team. Should new staff be recruited to the service, the original service delivery model would require to be reinstated. It is proposed that less experienced FES clinical services may benefit from maintaining the service delivery as per the model until such times. Provision of an annual review could be questioned. The annual review did however provide support, ensured compliance and allowed maintenance of optimum FES set-up and gave an overall re-evaluation of the patient's general condition. An additional value is that it is a method of meeting the National Stroke Guidelines, which recommend that patients with reduced ability six months poststroke should be routinely reassessed (Royal College of Physicians, 2008).

Subgroup analyses comparing those who had an AFO and those who had no orthotic device at assessment were undertaken. The no orthotic group walked faster at baseline, possibly indicating a higher level of mobility in this subgroup, and were only 2.2 years post-stroke compared to 5.4 years in the AFO subgroup at the time of assessment. Interestingly, at baseline, there was no statistically significant difference between groups for cadence. By six months, statistical analysis indicated that the no orthotic subgroup had significantly greater gait velocity and cadence in comparison to the AFO subgroup. It could be hypothesised that these greater improvements in the no orthotic group were achieved because, prior to attendance at the clinic, they had no other orthotic device for the correction of their dropped foot. However, it is of interest to note from this service evaluation that there was still a statistically significant benefit of FES application to patients in the AFO subgroup who already had an existing orthotic device. It could be suggested that those who already have an orthotic device should not demonstrate any significant improvements in cadence and gait velocity with FES, as it is another form of orthosis. One study comparing AFO, FES and no orthotic device for the correction of dropped foot following stroke concluded that FES and AFO improved functional ambulation above that of no device (Sheffler et al., 2006). The study authors reported that due to the study design they could not make direct comparisons on the superiority of one orthotic device over another. The National Guidelines for Stroke recommend the use of FES only if the AFO is not controlling the foot and if FES improves gait (Royal College of Physicians, 2008). In the current service evaluation, patients who attended at initial assessment with an AFO were seeking alternative devices for numerous reasons, that is, poor cosmesis, difficulties with footwear and discomfort. Although clinically the AFO was deemed to be adequately controlling the 
dropped foot, once fitted with FES, these patients demonstrated statistically significant improvements in their gait velocity and cadence. These results, while only a service evaluation, may bring the Royal College of Physicians recommendations into question. Further rigorous research directly comparing AFO and FES in the management of dropped foot in stroke is required, which also includes more detailed gait analysis and an economic evaluation of both modalities.

\section{Strengths and limitations}

By its nature, service evaluation lacks the validity and rigour of more scientific research (National Patient Safety Agency, 2009). It is therefore recognised that the transferability of these results may be limited. At the concept of the clinic, every effort was made by members of the clinical team to remain as neutral as possible, but they were an integral component of the service and therefore could be viewed as bias. Of the total cohort of 50 referred to the service, $20 \%$ were excluded for various reasons (progressive illness; recovery of movement; died; assessed as unsuitable; failed to attend; unable to cope with the device). This may be believed to have had an impact on the results and could be perceived as selection bias. However, the clinical outcomes were in line with current literature, and this could be considered to give the quantitative results some degree of external validity. There is always the question of whether a statistically significant result translates into a result of clinical significance. There is no clear documentation of what is a clinically significant change in stroke patients. Collen et al. (1990) stated that a significant difference in gait speed in stroke patients equated to a change of $25 \%$. In this study, on average, the total cohort improved its gait velocity by $0.2 \mathrm{~m} / \mathrm{s}$, which is a $39 \%$ increase. These results could therefore be considered as clinically significant.

Qualitative research must be examined for its credibility. Participants were identified from the clinic database by two members of the research team (JS; KW). This could be seen as selection bias. To address this issue, purposive sampling with a clear stratification process was applied by an independent researcher (CB). This aimed to ensure that a diverse clinic population was included. Data were collected by an independent researcher who had previous qualitative experience but no involvement in the clinic, which enhances the validity of the results. Participants were then free to express their views in relation to the clinic. All transcripts were transcribed verbatim by an independent research assistant and participant interview summary and research team code verification was used. This strengthens the rigour of these results. Owing to the nature of data collection methods in qualitative research, the views of those with communication difficulties are often excluded. This study recognised this limitation and attempted to address this issue by including carers of patients with marked communication difficulties, which potentially offers some insight into their perception of the clinic. By encompassing the views of patients and carers and by using the stratification process to get a diverse clinic population, the strength and transferability of these results are enhanced.

Practice reflection, by its nature, is subjective and encompasses the personal perspectives of the individual/individuals undertaking it. Others undertaking a reflection of the FES clinic may have different views and perceptions. The inclusion of this additional dimension in the service evaluation allowed the clinically relevant experiences of the clinicians on the value of the service delivery model and the clinical application to be included. This, combined with the qualitative and quantitative component of this study, provided a global review.

This study provides insights about the provision of an FES clinic to a specific population, namely chronic stroke patients. The requirements for other neurological populations, especially those with progressive illnesses, in relation to FES provision need to be investigated. This limits the generalisability of these results.

\section{Conclusion}

The results of this service evaluation would indicate that overall the pilot FES clinic design met the needs of chronic patients with stroke and carers with only minor modifications identified. In addition, in line with the current literature, it produced highly statistically significant improvements in physical outcomes comparing before and after FES application. Significant improvements in gait 
velocity and cadence were also demonstrated in a subgroup analysis of participants who used an AFO at initial assessment. The addition of qualitative research to this study ensured that the views of patients and carers were encompassed. This could be proposed as a credible method for achieving the aim of increasing carers and patients' involvement in NHS service development. This service evaluation potentially provides a documented and evidence- based clinical service delivery model for the provision of FES to chronic stroke patients that could be readily and widely clinically implemented and addresses the lack of clarity over the definition of a specialist team. The use of quantitative and qualitative evaluation and clinical reflection provided a global review of the service and suggestions for further research. The data gathered from this evaluation continue to be used to support the establishment and development of an optimum FES clinical service in Lothian.

\section{Outcome}

In 2008, a mainstream clinic 'The Lothian FES Service', assessing 24 patients per annum, was set up using resources from the Physiotherapy Neurological outpatients service. Service funding remains limited.

\section{Acknowledgements}

The authors wish to thank the patients attending the Functional electrical stimulation (FES) clinic, all participants who gave up their time to take part in the qualitative study and the Lothian Managed Clinical Network for stroke and Ecas for their financial support. In addition, the qualitative component of this study was supported by a pump-priming grant from the Centre for Integrated Healthcare Research. The authors also wish to thank Caroline McGuire for her contribution to the FES service design, provision and service evaluation and Judy Scopes, physiotherapy manager, for her support.

\section{References}

Burridge, J. 2001: Does the drop-foot stimulator improve walking in hemiplegia? Neuromodulation 4, 77-83.
Collen, F.M., Wade, D.T. and Bradshaw, C.M. 1990: Mobility after stroke: reliability of measures of impairment and disability. International Disability Studies 12, 6-9.

Department of Health. 2007: National stroke strategy. London: Department of Health.

Kottink, A., Ootendor, L., Buurke, J., Nene, A.V., Hermens, H.J. and Ijzerman, M.J. 2004: The orthotic effect of functional electrical stimulation on the improvement of walking in stroke patients with a dropped foot: a systematic review. Artificial Organs 28, 577-86.

Laufer, Y., Hausdorff, J.M. and Ring, H. 2009: Effects of a foot drop neuroprosthesis on functional abilities, social participation and gait velocity. American Journal of Physical Medicine and Rehabilitation 88, 14-20.

Mudge, S. and Stott, S.N. 2007: Outcome measures to assess walking ability following stroke: a systematic review of the literature. Physiotherapy 93, 189-200.

National Clinical FES Centre. Referral criteria and treatment protocol. Retrieved 16 January 2010 from http://www. salisburyfes.com/pdfs/referral.PDF

National Institute for Health and Clinical Excellence. 2009: Interventional procedure guidance 278: functional electrical stimulation for drop foot of central neurological origin. London: NICE.

National Patient Safety Agency. 2009: National research ethics service - defining research (reference 0987). London: National Patient Safety Agency.

Robbins, S.M., Houghton, P.E., Woodbury, M.G. and Brown, J.L. 2006: The therapeutic effect of functional and transcutaneous electric stimulation on improving gait speed in stroke patients: a meta-analysis. Archives of Physical Medicine and Rehabilitation 87, 853-59.

Royal College of Physicians. 2008: National guidelines for stroke, third edition. London: Royal College of Physicians.

Sheffler, L.R., Hennessey, M.T., Naples, G.G. and Chae, J. 2006: Peroneal nerve stimulation versus an ankle foot orthosis for correction of footdrop in stroke: impact on functional ambulation. Neurorehabilitation and Neural Repair 20, 355-60.

Smith, J. 2008: Qualitative psychology a practical guide to research methods, second edition. London: Sage Publications.

Taylor, P.N., Burridge, J.H., Dunkerley, A.L., Lamb, A., Wood, D. and Norton, J.A. 1999a: Patients perceptions of the Odstock Dropped Foot Stimulator (ODFS). Clinical Rehabilitation 13, 439-46.

Taylor, P., Burridge, J., Dunkerley, A., Wood, D., Norton, J., Singleton, C. and Swain, I. 1999b: Clinical audit of 5 years provision of the Odstock Dropped Foot Stimulator. Artificial Organs 23, 440-42.

Verdie, C., Daviet, J.C., Borie, M.J., Popielarz, S., Munoz, M., Salle, J.Y., Rebeyrotte, I. and Dudognon, P. 2004: Epidemiology of pes varus and/or equinus one year after a first cerebral hemisphere stroke: apropos of a cohort of 86 patients. Annales de réadaptation et de médecine physique 47, 81-86. 